\title{
Supplementary Information for
}

\section{DFT Estimate of Halide Perovskites Band Gap: When Spin Orbit Coupling}

\section{helps}

\author{
Tilak Das, Giovanni Di Liberto,* and Gianfranco Pacchioni \\ Dipartimento di Scienza dei Materiali, Università degli Studi Milano-Bicocca, \\ via R. Cozzi, 55 - 20125 Milano, Italy
}

Table S1: Crystal structure information of the 36 halide perovskites investigated here.

\begin{tabular}{|c|c|c|c|}
\hline N. & Materials & Space Group (Temperature) & Lattice Parameters \\
\hline 1 & $\alpha-\mathrm{CsPbCl}_{3}$ & $221(320 \mathrm{~K})$ & $a=5.605 \AA^{1-3}$ \\
\hline 2 & $\alpha-\mathrm{CsPbBr}_{3}$ & $221(403 \mathrm{~K})$ & $a=5.870 \AA^{4,5}$ \\
\hline 3 & $\gamma-\mathrm{CsPbBr}_{3}$ & $62(293 \mathrm{~K})$ & $a=8.244 \AA b=11.735 \AA c=8.198 \AA^{6}$ \\
\hline 4 & $\alpha-\mathrm{CsPbI}_{3}$ & $221(598 \mathrm{~K})$ & $a=6.289 \AA^{7}$ \\
\hline 5 & $\gamma-\mathrm{CsPbI}_{3}$ & $62(298 \mathrm{~K})$ & $a=8.856 \AA b=8.577 \AA c=12.472 \AA^{8}$ \\
\hline 6 & $\alpha-\mathrm{CsSnCl}_{3}$ & $221(390 \mathrm{~K})$ & $a=5.504 \AA$ \\
\hline 7 & $\alpha-\mathrm{CsSnBr}_{3}$ & $221(293 \mathrm{~K})$ & $a=5.804 \AA^{9,10}$ \\
\hline 8 & $\alpha-\mathrm{CsSnI}_{3}$ & $221(500 \mathrm{~K})$ & $a=6.206 \AA^{11}$ \\
\hline 9 & $\gamma-\mathrm{CsSnI}_{3}$ & $62(300 \mathrm{~K})$ & $a=8.689 \AA b=12.378 \AA c=8.638 \AA^{12}$ \\
\hline 10 & $\alpha-\mathrm{CsGeCl}_{3}$ & $221(428 \mathrm{~K})$ & $a=5.434 \AA^{13}$ \\
\hline 11 & $\alpha-\mathrm{CsGeBr}_{3}$ & $221(511 \mathrm{~K})$ & $a=5.636 \AA^{13}$ \\
\hline 12 & $\alpha-\mathrm{CsGeI}_{3}$ & $221(550 \mathrm{~K})$ & $a=5.983 \AA^{13}$ \\
\hline 13 & $\alpha-\mathrm{MAPbCl}_{3}$ & $221(298 \mathrm{~K})$ & $a=5.684 \AA^{14,15}$ \\
\hline 14 & $\alpha-\mathrm{MAPbBr}_{3}$ & $221(298 \mathrm{~K})$ & $a=5.931 \AA^{14}$ \\
\hline 15 & $\gamma-\mathrm{MAPbBr}_{3}$ & $33(145 \mathrm{~K})$ & $a=7.979 \AA b=8.580 \AA c=11.849 \AA^{16}$ \\
\hline 16 & $\alpha-\mathrm{MAPbI}_{3}$ & $221(298 \mathrm{~K})$ & $a=6.329 \AA^{14,17}$ \\
\hline 17 & $t$-MAPbI 3 & $108(162 \mathrm{~K})$ & $a=b=8.929 \AA c=12.688 \AA^{17,18}$ \\
\hline
\end{tabular}




\begin{tabular}{|c|c|c|c|}
\hline 18 & $\gamma-\mathrm{MAPbI}_{3}$ & $62(100 \mathrm{~K})$ & $a=8.856 \AA b=12.610 \AA c=8.575 \AA^{17,19}$ \\
\hline 19 & $\alpha-\mathrm{MASnCl}_{3}$ & $221(463 \mathrm{~K})$ & $a=5.760 \AA^{12}$ \\
\hline 20 & $\alpha-\mathrm{MASnBr}_{3}$ & $221(195 \mathrm{~K})$ & $a=5.899 \AA^{12}$ \\
\hline 21 & $\alpha-\mathrm{MASnI}_{3}$ & $221(298 \mathrm{~K})$ & $a=6.239 \AA^{20}$ \\
\hline 22 & $t-\mathrm{MASnI}_{3}$ & $140(140 \mathrm{~K})$ & $a=b=8.727 \AA c=12.502 \AA^{21}$ \\
\hline 23 & $\gamma$-MASnI 3 & $62(110 \mathrm{~K})$ & $a=8.745 \AA b=12.353 \AA c=8.526 \AA^{21}$ \\
\hline 24 & $\alpha-\mathrm{MAGeBr}_{3}$ & $221(298 \mathrm{~K})$ & $a=5.891 \AA^{22}$ \\
\hline 25 & $\alpha-\mathrm{MAGeI}_{3}$ & $221(293 \mathrm{~K})$ & $a=6.048 \AA^{23,24}$ \\
\hline 26 & $\alpha-\mathrm{FAPbCl}_{3}$ & $221(298 \mathrm{~K})$ & $a=5.800 \AA^{25}$ \\
\hline 27 & $\alpha-\mathrm{FAPbBr}_{3}$ & $221(298 \mathrm{~K})$ & $a=6.000 \AA^{26}$ \\
\hline 28 & $t$-FAPbBr 3 & $127(250 \mathrm{~K})$ & $a=b=8.415 \AA c=5.947 \AA^{27}$ \\
\hline 29 & $\gamma-\mathrm{FAPbBr}_{3}$ & $62(125 \mathrm{~K})$ & $a=8.369 \AA b=11.831 \AA c=8.360 \AA^{28}$ \\
\hline 30 & $\alpha-\mathrm{FAPbI}_{3}$ & $221(298 \mathrm{~K})$ & $a=6.362 \AA^{29}$ \\
\hline 31 & $\alpha-\mathrm{FASnBr}_{3}$ & $221(298 \mathrm{~K})$ & $a=6.056 \AA^{30}$ \\
\hline 32 & $\alpha-\mathrm{FASnI}_{3}$ & $221(298 \mathrm{~K})$ & $a=6.316 \AA^{31}$ \\
\hline 33 & $t$-FASnI 3 & $100(155 \mathrm{~K})$ & $a=b=8.838 \AA c=12.406 \AA^{32}$ \\
\hline 34 & $\gamma$-FASnI 3 & $62(125 \mathrm{~K})$ & $a=8.8175 \AA b=12.4164 \AA c=8.8578 \AA^{27}$ \\
\hline 35 & $t-\mathrm{CsPbBr}_{3}$ & $106(361 \mathrm{~K})$ & $a=b=11.675 \AA c=11.627 \AA^{33,34}$ \\
\hline 36 & $t$-MAPbBr 3 & $140(155 \mathrm{~K})$ & $a=b=8.322 \AA c=11.832 \AA^{16}$ \\
\hline
\end{tabular}

\section{S1 Dielectric dependent HSE06 band gaps of halide perovskites}

The optimal exact Fock exchange fraction $(\alpha)$ for a given compound can be obtained self-consistently by converging the dielectric constant $\left(\varepsilon_{\infty}\right)$, and defining at each time $\alpha=1 / \varepsilon_{\infty}{ }^{35-38}$ We selected a subset of 14 halide perovskites and we first determined self-consistently the optimal $\alpha$, then we calculated the band gap and both HSE06 and HSE06/SOC levels. The calculations have been restricted to a small set of data because of the high computational demand required for converging $\alpha$. Table S2 shows the calculated band gaps compared with the experimental ones. 
Table S2: Calculated band gap (in eV) from dielectric-dependent (DD) HSE06. The calculations are done with and without SOC.

\begin{tabular}{|c|c|c|c|c|c|}
\hline Materials & $\alpha$ & HSE06 & $\alpha$ & $(\mathrm{HSE06}+\mathrm{SOC})_{\mathrm{DD}}$ & Exp. $^{\text {av }}$ \\
\hline$\alpha-\mathrm{CsPbCl}_{3}$ & 0.35 & 3.80 & 0.43 & 3.18 & 3.09 \\
\hline$\alpha-\mathrm{CsPbBr}_{3}$ & 0.30 & 2.97 & 0.33 & 2.07 & 2.36 \\
\hline$\gamma-\mathrm{CspbBr}_{3}$ & 0.30 & 3.36 & 0.36 & 2.60 & 2.37 \\
\hline$\alpha-\mathrm{CsPbI}_{3}$ & 0.23 & 2.33 & 0.20 & 1.04 & 1.81 \\
\hline$\gamma-\mathrm{CsPbI}_{3}$ & 0.24 & 2.74 & 0.26 & 1.79 & 2.12 \\
\hline$\alpha-\mathrm{CsSnCl}_{3}$ & 0.25 & 1.76 & 0.30 & 1.65 & 2.85 \\
\hline$\alpha-\mathrm{CsSnBr}_{3}$ & 0.18 & 1.16 & 0.19 & 0.82 & 1.84 \\
\hline$\gamma-\mathrm{CsSnI}_{3}$ & 0.16 & 1.14 & 0.17 & 0.82 & 1.31 \\
\hline$\alpha-\mathrm{CsGeCl}_{3}$ & 0.28 & 2.75 & 0.35 & 3.00 & 3.55 \\
\hline$\alpha-\mathrm{CsGeBr}_{3}$ & 0.19 & 1.69 & 0.22 & 1.67 & 2.15 \\
\hline$\alpha-\mathrm{CsGeI}_{3}$ & 0.11 & 1.02 & 0.10 & 0.73 & 1.67 \\
\hline$\alpha-\mathrm{MAPbCl}_{3}$ & 0.37 & 4.21 & 0.37 & 3.07 & 3.02 \\
\hline$\alpha-\mathrm{MAPbBr}_{3}$ & 0.30 & 3.23 & 0.28 & 1.98 & 2.28 \\
\hline$\alpha-\mathrm{MAPbI}_{3}$ & 0.23 & 2.40 & 0.18 & 0.99 & 1.62 \\
\hline MAE & & 0.73 & & 0.52 & \\
\hline
\end{tabular}




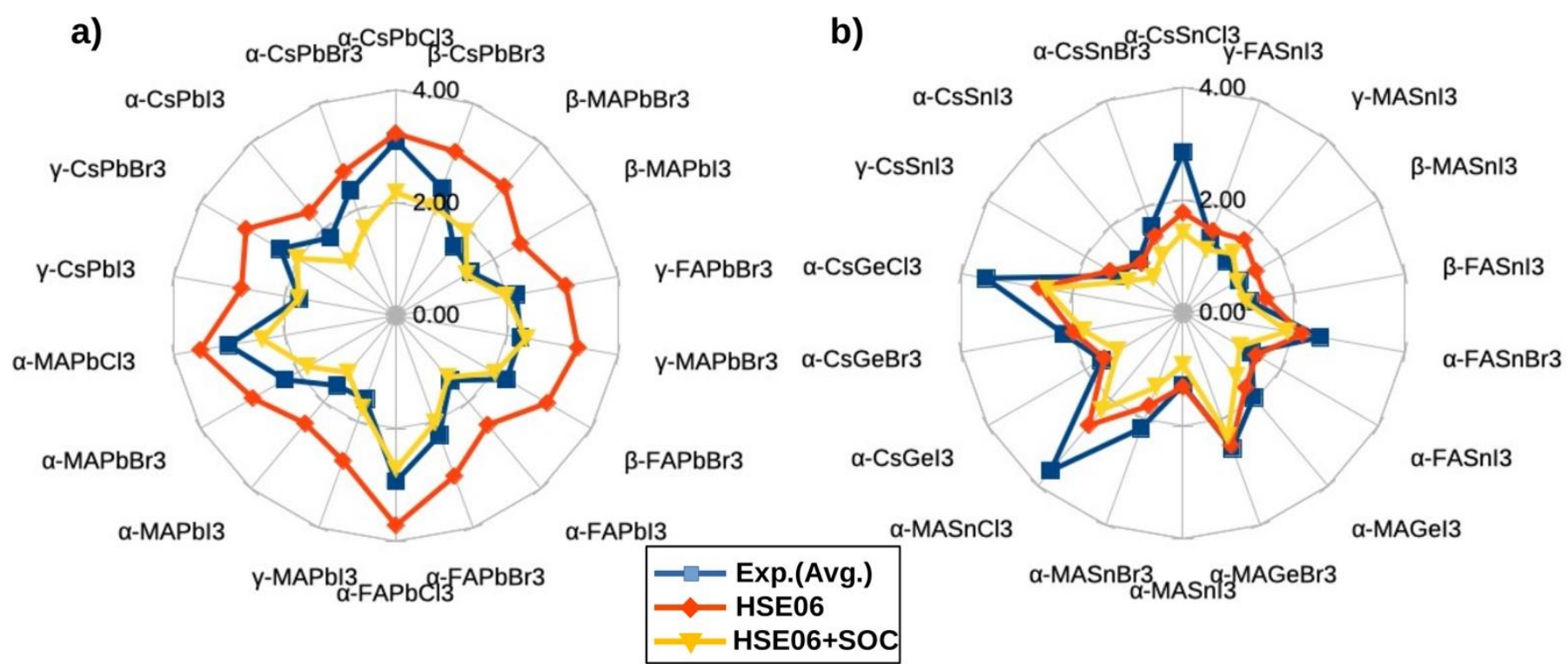

Figure S1 Graph of band gaps of Pb-based (panel a) and Pb-free (panel b) halide perovskites. Blue line is for Experimental values, red line is for HSE06, and yellow line is for HSE06/SOC.

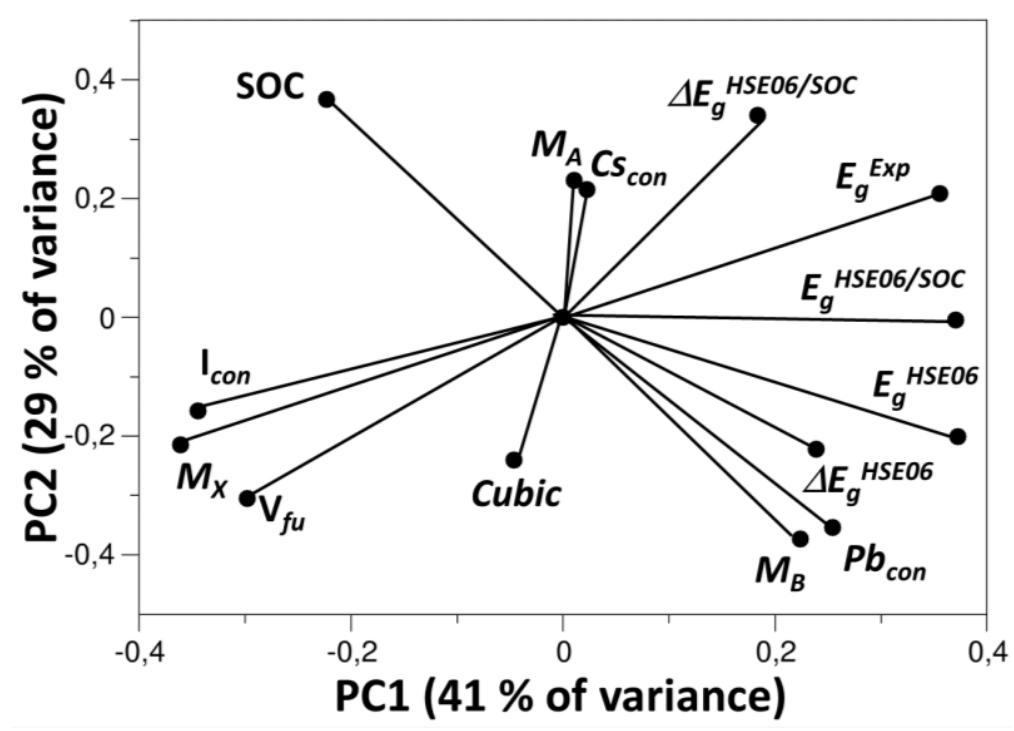

Figure S2: Loading plot made by the main principal components (PC1, PC2). The original variables are reported as vectors in the new reference frame. 


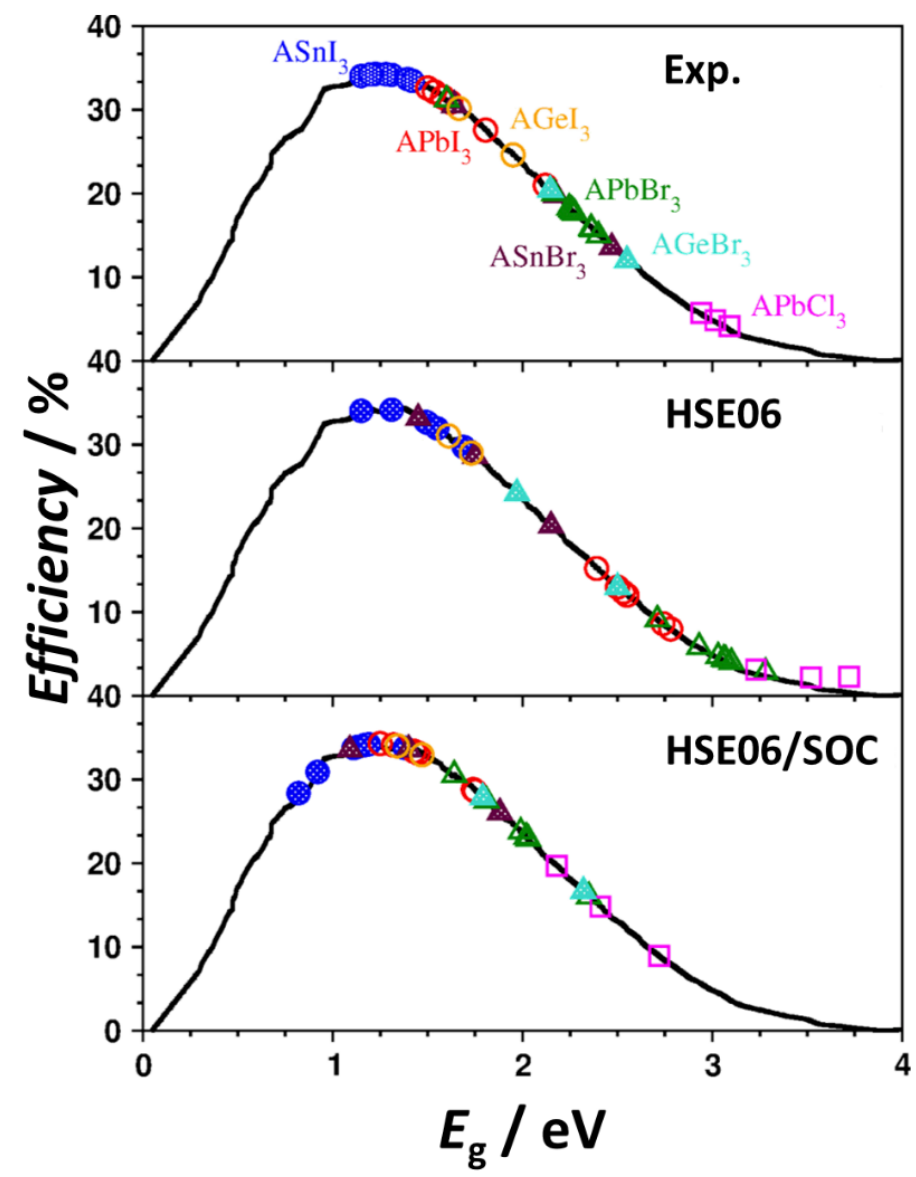

Figure S3: Efficiency vs band gap of 36 halide perovskites based on Shockley-Queisser detailed balance approach; ${ }^{39}$ (b) The same plot but by using HSE06 and (c) HSE06/SOC calculated band gaps. Circles: Iodides; triangles: Bromides; squares: Chlorides; empty contour: Pb-based materials; solid contour: Sn-based materials; dotted contour: Ge-based materials.

\section{References}

(1) MØLLER, C. K. A Phase Transition in Cæsium Plumbochloride. Nature 1957, 180 (4593), 981-982. https://doi.org/10.1038/180981a0.

(2) MØLLER, C. K. Crystal Structure and Photoconductivity of Cæsium Plumbohalides. Nature 1958, 182 (4647), 1436-1436. https://doi.org/10.1038/1821436a0.

(3) Hutton, J.; Nelmes, R. J.; Meyer, G. M.; Eiriksson, V. R. High-Resolution Studies of Cubic Perovskites by Elastic Neutron Diffraction: $\mathrm{CsPbCl}$ 3. J. Phys. C Solid State Phys. 1979, 12 (24), 5393-5410. https://doi.org/10.1088/0022-3719/12/24/011.

(4) No Title. https://doi.org/10.1023/A:1022836800820.

(5) Sakata, M.; Nishiwaki, T.; Harada, J. Neutron Diffraction Study of the Structure of Cubic CsPbBr 3. J. Phys. Soc. Japan 1979, 47 (1), 232-233. https://doi.org/10.1143/JPSJ.47.232.

(6) Stoumpos, C. C.; Malliakas, C. D.; Peters, J. A.; Liu, Z.; Sebastian, M.; Im, J.; Chasapis, T. C.; Wibowo, A. C.; Chung, D. Y.; Freeman, A. J.; et al. Crystal Growth of the Perovskite 
Semiconductor $\mathrm{CsPbBr} 3$ : A New Material for High-Energy Radiation Detection. Cryst. Growth Des. 2013, 13 (7), 2722-2727. https://doi.org/10.1021/cg400645t.

(7) Sharma, S.; Weiden, N.; Weiss, A. Phase Diagrams of Quasibinary Systems of the Type: $\mathrm{ABX} 3-\mathrm{A}^{\prime} \mathrm{BX} 3$; $\mathrm{ABX} 3-\mathrm{AB}^{\prime} \mathrm{X} 3$, and $\mathrm{ABX} 3-\mathrm{ABX}^{\prime} 3$; X = Halogen. Zeitschrift für Phys. Chemie 1992, 175 (Part_1), 63-80. https://doi.org/10.1524/zpch.1992.175.Part_1.063.

(8) Sutton, R. J.; Filip, M. R.; Haghighirad, A. A.; Sakai, N.; Wenger, B.; Giustino, F.; Snaith, H. J. Cubic or Orthorhombic? Revealing the Crystal Structure of Metastable Black-Phase CsPbI 3 by Theory and Experiment. ACS Energy Lett. 2018, 3 (8), 1787-1794. https://doi.org/10.1021/acsenergylett.8b00672.

(9) Barrett, J.; Bird, S. R. A.; Donaldson, J. D.; Silver, J. The Mössbauer Effect in Tin( $<\mathrm{scp}>\mathrm{II}</$ Scp $>$ ) Compounds. Part XI. The Spectra of Cubic Trihalogenostannates( <scp>II</Scp> ). J. Chem. Soc. A 1971, 3105-3108. https://doi.org/10.1039/J19710003105.

(10) Mori, M.; Saito, H. An X-Ray Study of Successive Phase Transitions in CsSnBr 3. J. Phys. C Solid State Phys. 1986, 19 (14), 2391-2401. https://doi.org/10.1088/0022-3719/19/14/005.

(11) Chung, I.; Song, J.-H.; Im, J.; Androulakis, J.; Malliakas, C. D.; Li, H.; Freeman, A. J.; Kenney, J. T.; Kanatzidis, M. G. CsSnI 3 : Semiconductor or Metal? High Electrical Conductivity and Strong Near-Infrared Photoluminescence from a Single Material. High Hole Mobility and Phase-Transitions. J. Am. Chem. Soc. 2012, 134 (20), 8579-8587. https://doi.org/10.1021/ja301539s.

(12) Baikie, T.; Fang, Y.; Kadro, J. M.; Schreyer, M.; Wei, F.; Mhaisalkar, S. G.; Graetzel, M.; White, T. J. Synthesis and Crystal Chemistry of the Hybrid Perovskite (CH3NH3)PbI3 for Solid-State Sensitised Solar Cell Applications. J. Mater. Chem. A 2013, 1 (18), 5628. https://doi.org/10.1039/c3ta10518k.

(13) Tang, L.-C.; Chang, Y.-C.; Huang, J.-Y.; Lee, M.-H.; Chang, C.-S. First Principles Calculations of Linear and Second-Order Optical Responses in Rhombohedrally Distorted Perovskite Ternary Halides, CsGeX 3 (X = Cl, Br, and I). Jpn. J. Appl. Phys. 2009, 48 (11), 112402. https://doi.org/10.1143/JJAP.48.112402.

(14) Baikie, T.; Barrow, N. S.; Fang, Y.; Keenan, P. J.; Slater, P. R.; Piltz, R. O.; Gutmann, M.; Mhaisalkar, S. G.; White, T. J. A Combined Single Crystal Neutron/X-Ray Diffraction and Solid-State Nuclear Magnetic Resonance Study of the Hybrid Perovskites CH 3 NH 3 PbX 3 (X = I, Br and Cl). J. Mater. Chem. A 2015, 3 (17), 9298-9307. https://doi.org/10.1039/C5TA01125F.

(15) Sektiono, M. W. A.; Permatasari, F. A.; Aimon, A. H.; Iskandar, F. Rapid Growth of the CH $3 \mathrm{NH} 3 \mathrm{PbCl} 3$ Single Crystal by Microwave Irradiation. RSC Adv. 2021, 11 (3), 1360-1366. https://doi.org/10.1039/D0RA09328A.

(16) Poglitsch, A.; Weber, D. Dynamic Disorder in Methylammoniumtrihalogenoplumbates (II) Observed by Millimeter-wave Spectroscopy. J. Chem. Phys. 1987, 87 (11), 6373-6378. https://doi.org/10.1063/1.453467.

(17) Oku, T. Crystal Structures of Perovskite Halide Compounds Used for Solar Cells. Rev. Adv. Mater. Sci. 2020, 59 (1), 264-305. https://doi.org/10.1515/rams-2020-0015.

(18) Hao, F.; Stoumpos, C. C.; Chang, R. P. H.; Kanatzidis, M. G. Anomalous Band Gap Behavior in Mixed $\mathrm{Sn}$ and $\mathrm{Pb}$ Perovskites Enables Broadening of Absorption Spectrum in Solar Cells. J. Am. Chem. Soc. 2014, 136 (22), 8094-8099. https://doi.org/10.1021/ja5033259. 
(19) Whitfield, P. S.; Herron, N.; Guise, W. E.; Page, K.; Cheng, Y. Q.; Milas, I.; Crawford, M. K. Structures, Phase Transitions and Tricritical Behavior of the Hybrid Perovskite Methyl Ammonium Lead Iodide. Sci. Rep. 2016, 6 (1), 35685. https://doi.org/10.1038/srep35685.

(20) Mitzi, D. B.; Feild, C. A.; Schlesinger, Z.; Laibowitz, R. B. Transport, Optical, and Magnetic Properties of the Conducting Halide Perovskite CH3NH3SnI3. J. Solid State Chem. 1995, 114 (1), 159-163. https://doi.org/10.1006/jssc.1995.1023.

(21) Takahashi, Y.; Obara, R.; Lin, Z.-Z.; Takahashi, Y.; Naito, T.; Inabe, T.; Ishibashi, S.; Terakura, K. Charge-Transport in Tin-Iodide Perovskite CH3NH3SnI3: Origin of High Conductivity. Dalt. Trans. 2011, 40 (20), 5563. https://doi.org/10.1039/c0dt01601b.

(22) Chang, X.; Marongiu, D.; Sarritzu, V.; Sestu, N.; Wang, Q.; Lai, S.; Mattoni, A.; Filippetti, A.; Congiu, F.; Lehmann, A. G.; et al. Layered Germanium Hybrid Perovskite Bromides: Insights from Experiments and First-Principles Calculations. Adv. Funct. Mater. 2019, 29 (31), 1903528. https://doi.org/10.1002/adfm.201903528.

(23) Stoumpos, C. C.; Frazer, L.; Clark, D. J.; Kim, Y. S.; Rhim, S. H.; Freeman, A. J.; Ketterson, J. B.; Jang, J. I.; Kanatzidis, M. G. Hybrid Germanium Iodide Perovskite Semiconductors: Active Lone Pairs, Structural Distortions, Direct and Indirect Energy Gaps, and Strong Nonlinear Optical Properties. J. Am. Chem. Soc. 2015, 137 (21), 6804-6819. https://doi.org/10.1021/jacs.5b01025.

(24) Wu, L.; Lu, P.; Li, Y.; Sun, Y.; Wong, J.; Yang, K. First-Principles Characterization of TwoDimensional (CH 3 (CH 2 ) 3 NH 3 ) 2 ( $\mathrm{CH} 3$ NH 3 ) N-1 Ge n I 3n+1 Perovskite. J. Mater. Chem. A 2018, 6 (47), 24389-24396. https://doi.org/10.1039/C8TA10055A.

(25) Levchuk, I.; Osvet, A.; Tang, X.; Brandl, M.; Perea, J. D.; Hoegl, F.; Matt, G. J.; Hock, R.; Batentschuk, M.; Brabec, C. J. Brightly Luminescent and Color-Tunable Formamidinium Lead Halide Perovskite FAPbX 3 (X = Cl, Br, I) Colloidal Nanocrystals. Nano Lett. 2017, 17 (5), 2765-2770. https://doi.org/10.1021/acs.nanolett.6b04781.

(26) Jang, D. M.; Kim, D. H.; Park, K.; Park, J.; Lee, J. W.; Song, J. K. Ultrasound Synthesis of Lead Halide Perovskite Nanocrystals. J. Mater. Chem. C 2016, 4 (45), 10625-10629. https://doi.org/10.1039/C6TC04213A.

(27) Schueller, E. C.; Laurita, G.; Fabini, D. H.; Stoumpos, C. C.; Kanatzidis, M. G.; Seshadri, R. Crystal Structure Evolution and Notable Thermal Expansion in Hybrid Perovskites Formamidinium Tin Iodide and Formamidinium Lead Bromide. Inorg. Chem. 2018, 57 (2), 695-701. https://doi.org/10.1021/acs.inorgchem.7b02576.

(28) Franz, A.; Többens, D. M.; Lehmann, F.; Kärgell, M.; Schorr, S. The Influence of Deuteration on the Crystal Structure of Hybrid Halide Perovskites: A TemperatureDependent Neutron Diffraction Study of FAPbBr 3. Acta Crystallogr. Sect. B Struct. Sci. Cryst. Eng. Mater. 2020, 76 (2), 267-274. https://doi.org/10.1107/S2052520620002620.

(29) Weller, M. T.; Weber, O. J.; Frost, J. M.; Walsh, A. Cubic Perovskite Structure of Black Formamidinium Lead Iodide, $\alpha-[\mathrm{HC}(\mathrm{NH} 2$ ) 2 ]PbI 3 , at 298 K. J. Phys. Chem. Lett. 2015, 6 (16), 3209-3212. https://doi.org/10.1021/acs.jpclett.5b01432.

(30) Yamada, K.; Fujise, K.; Hino, S.; Yamane, Y.; Nakagama, T. Characterization of Sn(II)Based Perovskites by XRD, DTA, NQR and 119 Sn NMR for Photovoltaic Applications. Chem. Lett. 2019, 48 (7), 749-752. https://doi.org/10.1246/cl.190262.

(31) No Title. https://doi.org/10.1006/jssc.1997.7593Get. 
(32) Kahmann, S.; Nazarenko, O.; Shao, S.; Hordiichuk, O.; Kepenekian, M.; Even, J.; Kovalenko, M. V.; Blake, G. R.; Loi, M. A. Negative Thermal Quenching in FASnI 3 Perovskite Single Crystals and Thin Films. ACS Energy Lett. 2020, 5 (8), 2512-2519. https://doi.org/10.1021/acsenergylett.0c01166.

(33) Hirotsu, S.; Harada, J.; Iizumi, M.; Gesi, K. Structural Phase Transitions in CsPbBr 3. J. Phys. Soc. Japan 1974, 37 (5), 1393-1398. https://doi.org/10.1143/JPSJ.37.1393.

(34) Xue, J.; Yang, D.; Cai, B.; Xu, X.; Wang, J.; Ma, H.; Yu, X.; Yuan, G.; Zou, Y.; Song, J.; et al. Photon-Induced Reversible Phase Transition in $\mathrm{CsPbBr} 3$ Perovskite. Adv. Funct. Mater. 2019, 29 (13), 1807922. https://doi.org/10.1002/adfm.201807922.

(35) Gerosa, M.; Bottani, C. E.; Caramella, L.; Onida, G.; Di Valentin, C.; Pacchioni, G. Electronic Structure and Phase Stability of Oxide Semiconductors: Performance of Dielectric-Dependent Hybrid Functional DFT, Benchmarked against GW Band Structure Calculations and Experiments. Phys. Rev. B - Condens. Matter Mater. Phys. 2015, 91 (15), 1-15. https://doi.org/10.1103/PhysRevB.91.155201.

(36) Gerosa, M.; Bottani, C. E.; Di Valentin, C.; Onida, G.; Pacchioni, G. Accuracy of DielectricDependent Hybrid Functionals in the Prediction of Optoelectronic Properties of Metal Oxide Semiconductors: A Comprehensive Comparison with Many-Body GW and Experiments. $J$. Phys. Condens. Matter 2018, 30 (4), 044003. https://doi.org/10.1088/1361-648X/aa9725.

(37) Alkauskas, A.; Broqvist, P.; Pasquarello, A. Defect Levels through Hybrid Density Functionals: Insights and Applications. Phys. status solidi 2011, 248 (4), 775-789. https://doi.org/10.1002/pssb.201046195.

(38) Skone, J. H.; Govoni, M.; Galli, G. Self-Consistent Hybrid Functional for Condensed Systems. Phys. Rev. B 2014, 89 (19), 195112. https://doi.org/10.1103/PhysRevB.89.195112.

(39) Shockley, W.; Queisser, H. J. Detailed Balance Limit of Efficiency of P-n Junction Solar Cells. J. Appl. Phys. 1961, 32 (3), 510-519. https://doi.org/10.1063/1.1736034. 